\title{
MicroRNA-155 promotes tumor growth of human hepatocellular carcinoma by targeting ARID2
}

\author{
LI ZHANG ${ }^{1}$, WEI WANG ${ }^{2}$, XIAOBIN LI $^{2}$, SAI HE $^{3}$, JIANFENG YAO $^{4}$, \\ XIAOLONG WANG ${ }^{2}$, DI ZHANG ${ }^{1}$ and XUEJUN SUN ${ }^{2}$
}

\author{
${ }^{1}$ Department of General Surgery, The Second Affiliated Hospital of Xi'an Jiaotong University, Xi'an, \\ Shaanxi 710004; ${ }^{2}$ Department of General Surgery, The First Affiliated Hospital of Xi'an Jiaotong University; \\ ${ }^{3}$ Breast Cancer Program, Shaanxi Provincial Tumor Hospital, Xi'an, Shaanxi 710061; ${ }^{4}$ Department \\ of General Surgery, Shanxi Province People's Hospital, Xi'an, Shaanxi 710068, P.R. China
}

Received January 22, 2016; Accepted March 7, 2016

DOI: $10.3892 / \mathrm{ijo} .2016 .3465$

\begin{abstract}
Aberrant expression of microRNA-155 (miR-155) has been reported in several human cancers and is associated with prognosis of patients. However, the clinical significance of miR-155 and its underlying mechanisms involved in hepatocarcinogenesis remain to be determined. In this study, we demonstrated that the expression of miR-155 was elevated in both hepatocellular carcinoma (HCC) tissues and cell lines. Clinical association analysis revealed that high expression of miR-155 was correlated with malignant clinicopathological characteristics including large tumor size, high EdmondsonSteiner grading and TNM tumor stage. Furthermore, its high expression conferred a reduced 5-year overall survival and disease-free survival of HCC patients. Gain- and loss-of function studies revealed that miR-155 promoted cell cycle progression, cell proliferation and inhibited apoptosis. Mechanistically, we identified AT-rich interactive domain 2 (ARID2) as a direct downstream target and functional mediator of miR-155 in HCC cells. Notably, alterations of ARID2 expression abrogated the effects of miR-155 on HCC cell proliferation, cell cycle and apoptosis. Moreover, we demonstrated that Akt phosphorylation is essential for the functional roles of miR-155 through altering Cyclin D1 and p27, which were key components of cell cycle machinery. Finally, we disclosed that the downregulation of miR-155 suppressed tumor growth of HCC by inhibiting Akt signaling pathway. In conclusion, our results indicate that miR-155 promotes
\end{abstract}

Correspondence to: Dr Xuejun Sun, Department of General Surgery, The First Affiliated Hospital of Xi'an Jiaotong University, 277 Yanta West Road, Xi'an, Shaanxi 710061, P.R. China

E-mail: sunxj1234@sina.com

Dr Li Zhang, Department of General Surgery, The Second Affiliated Hospital of Xi'an Jiaotong University, 157 Xiwu Road, Xi'an, Shaanxi 710004, P.R. China

E-mail: z16403222@sina.com

Key words: miR-155, hepatocellular carcinoma, AT-rich interactive domain 2 , proliferation, cell cycle, apoptosis tumor growth of HCC by targeting ARID2-mediated Akt phosphorylation pathway, and potentially serves as a novel prognostic biomarker and therapeutic target for HCC.

\section{Introduction}

Hepatocellular carcinoma (HCC) is the dominant pathologic type of primary liver cancer, which is the third leading cause of cancer-related death worldwide (1). Despite recent advances in the diagnosis and treatment of $\mathrm{HCC}$, including hepatectomy and liver transplantation, the prognosis for HCC patients remains poor due to the high recurrence rate and early metastasis (2). However, the detailed mechanism underlying the development and progression of $\mathrm{HCC}$ is still not clear (3). Therefore, it is urgent to clarify the molecular mechanisms of HCC and identify novel prognostic biomarkers to provide potential therapeutic targets for the patients with HCC.

Increasing evidence suggests that microRNAs (miRNAs), a class of non-coding RNAs composed of $\sim 22$ nucleotides, can act as suitable biomarkers with diagnostic, prognostic, and predictive potential (4). miRNAs may function as oncogenes or tumor suppressors by regulating protein expression by interacting with complementary sites within the 3'-untranslated region (UTR) of target mRNA transcripts (5). Emerging studies have documented that aberrant miRNAs play important roles in various biological processes (6), including cell proliferation, differentiation, apoptosis, drug-resistance, migration and invasion (7-10). Recently, miRNAs have shown high stability in tissues and body fluids, which reveal their potential as tumor markers. In addition, it has been widely recognized that dysregulation of miRNAs contribute to the development and progression of HCC.

miR-155 is located within a region known as B cell integration cluster on chromosome 21 , which plays a critical role in the progression of gastric cancer (11), colorectal carcinoma (12), lung cancer (13), breast cancer (14), bladder cancer (15) and B-cell lymphoma (16). Previous studies show that miR-155 functions as a regulator in the pathogenesis of cell proliferation, apoptosis, drug-resistance, migration, invasion and epithelial-mesenchymal transition (EMT). miR-155 promotes oral squamous cell carcinoma metastasis and correlates a poor 
prognosis (17). miR-155 promotes the B-cell lymphoma cell proliferation and inhibits cell apoptosis by targeting NIAM phenocopies (16). Moreover, direct quantitative detection for cell-free miR-155 in urine could be a potential novel biomarker in diagnosis and prognosis for non-muscle invasive bladder cancer (15). miR-155 mediates anti-Warburg effect of rosmarinic acid in colorectal carcinoma and gastric cancer (18). miR-155 presents tamoxifen-resistance by modulating SOCS6STAT3 signaling pathway in breast cancer (19). However, the clinical significance of miR-155 and the underlying mechanisms involved in the development of HCC remain to be investigated.

In this study, we demonstrated that the expression of miR-155 was upregulated in HCC tissues and its high expression was associated with poor clinicopathological features and the reduced survival of HCC patients. miR-155 promoted cell proliferation, cell cycle and apoptosis resistance in vitro. Moreover, the downregulation of miR-155 inhibited tumor growth of HCC in vivo. Notably, AT-rich interactive domain 2 (ARID2) was identified as a direct target of miR-155. The results showed a new role for miR-155 in prediction of prognosis and promoting tumor growth of HCC.

\section{Materials and methods}

Clinical samples and cell lines. HCC samples (124) and matched normal tumor-adjacent samples $(>2 \mathrm{~cm}$ distance from the margin of the resection) were obtained during surgery and used after obtaining informed consent. All patients underwent resection of their primary HCC in the Department of General Surgery at The Second Affiliated Hospital of Xi'an Jiaotong University, from January 2006 to December 2008. None of the patients received preoperative chemo- or radiotherapy. The stage of cancer was determined according to the cancer staging system published in 2010 by the Union for International Cancer Control (UICC). The Xi'an Jiaotong University Ethics Committee approved all protocols according to the 1975 Helsinki Declaration.

Human HCC cell lines (Hep3B, Bel-7402, MHCC-97L, HepG2, SMMC-7721) and human immortalized normal hepatic cell line LO2 were obtained from the Institute of Biochemistry and Cell Biology, Chinese Academy of Sciences (Shanghai, China). The cells were maintained in Dulbecco's modified Eagle's medium (DMEM, Gibco, Grand Island, NY, USA) containing $10 \%$ fetal bovine serum (FBS, Gibco) with $100 \mathrm{U} / \mathrm{ml}$ penicillin and $100 \mu \mathrm{g} / \mathrm{ml}$ streptomycin (Sigma, St. Louis, MO, USA) and cultured in a humidified $5 \% \mathrm{CO}_{2}$ incubator at $37^{\circ} \mathrm{C}$.

Real-time quantitative reverse transcription polymerase chain reaction ( $q R T-P C R)$. Total RNA was extracted from clinical specimens or HCC cells using TRIzol reagent (Invitrogen, Carlsbad, CA, USA) according to the manufacturer's instructions. cDNA was synthesized from $1 \mu \mathrm{g}$ RNA with the PrimeScript RT Master Mix (Takara, Osaka, Japan). The PCR amplification for the quantification of the miR-155 and U6 was performed using the TaqMan miRNA Reverse Transcription kit (Applied Biosystems, Foster City, CA, USA) and TaqMan Human miRNA Assay kit (Applied Biosystems). The relative expression of miR-155 was shown as the fold difference relative to U6. qPCR primer against mature miRNA miR-155 (HmiRQP0221) and Homo sapiens snRNA U6 qPCR Primer (HmiRQP9001) were purchased from GeneCopoeia (Guangzhou, China).

Western blot analysis. Total protein was extracted from whole cells and $40 \mu \mathrm{g}$ of isolated protein was separated by $10 \%$ SDS-PAGE and transferred onto a PVDF membrane (Bio-Rad Laboratories, Hercules, CA, USA). The membranes were probed with antibodies: anti-Akt, anti-p-Akt, anti-ARID2, anti-Cyclin D, and anti-p27 primary antibodies (Cell Signaling, Danvers, MA, USA) overnight. Then the membranes were incubated with the HRP-conjugated goat anti-mouse or anti-rabbit IgG antibody (ZSGB-BIO, China). Protein bands were visualized using an enhanced chemiluminescence kit (Amersham, Little Chalfont, UK).

Immunohistochemical staining. Immunohistochemistry was performed on paraformaldehyde-fixed paraffin sections. ARID2 (1:100, \#13594 Cell Signaling Technology, Inc.) antibody was used in immunohistochemistry by a streptavidin peroxidase-conjugated (SP-IHC) method. The percentage of positive tumor cells was graded as: $0,<10 \% ; 1,10-30 \%$; $2,31-50 \% ; 3,>50 \%$.

Plasmids and cell transfection. miRNA vectors, including miR-155 expression vector (HmiR0358-MR02), the control vector for miR-155 (CmiR0001-MR04 and miR-control), miR-155 inhibitor (HmiR-AN0220-AM03 and anti-miR-155) and the negative control for the miR-155 inhibitor (CmiR-AN0001-AM04 and anti-miR-NC), and ARID2 expression plasmid were purchased from GeneCopoeia. The targeted sequences for ARID2 siRNA sense, 5'-AGCT CCAATTCCTTGTGAAGTTTT-3' and antisense, 5'-ACTTCA CAAGGAATTGCAGCTTTT-3' or a non-specific duplex oligonucleotide as a negative control were produced by Sangon Biotech Co., Ltd. (Shanghai, China). The cells were transfected with the vectors mentioned above using Lipofectamine 2000 according to the manufacturer's instructions (Invitrogen).

Cell cycle, proliferation and detection of apoptosis. Flow cytometry was performed using the fluorescence-activated cell sorting (FACS) Calibur and CellQuest software (both from Becton-Dickinson, San Jose, CA, USA). For cell cycle analysis, the cells were seeded in 6 -well plates at $2 \times 10^{5} /$ well. Forty-eight hours after transfection, the cells were fixed in $70 \%$ ethanol at $4^{\circ} \mathrm{C}$ for $24 \mathrm{~h}$ and stained with $50 \mu \mathrm{g} / \mathrm{ml}$ propidium iodide (Keygen, Nanjing, China). An Annexin V-Fluor Staining kit (Roche) was used to analyze apoptosis levels. For the proliferation assay, bromodeoxyuridine labeling and immunofluorescence was used. Cells grown on coverslips (Fisher, Pittsburgh, PA, USA) were incubated with bromodeoxyuridine (BrdU) for $1 \mathrm{~h}$ and stained with anti-BrdU antibody (Sigma) according to the manufacturer's instructions. Gray level images were acquired under a laser scanning microscope (Axioskop 2 plus, Carl Zeiss Co. Ltd., Jena, Germany).

Luciferase reporter assay. The 3'-UTR sequence of ARID2 predicted to interact with miR-155 or the mutated sequence within the predicted target sites was synthesized and inserted into the pGL3 control vector (Promega, Madison, WI, USA). These constructs were named as wt ARID2-3'UTR 
Table I. Clinical correlation of miR-155 expression in $\mathrm{HCC}$ $(n=124)$.

\begin{tabular}{lcccc}
\hline & & \multicolumn{2}{c}{ Expression level } & \\
Clinical & Cases & miR-155 & \\
parameters & $(\mathrm{n})$ & $(\mathrm{n}=68)$ & $\begin{array}{c}\text { miR-155 } \\
(\mathrm{n}=56)\end{array}$ & $\begin{array}{c}\text { P-value } \\
(\mathrm{p}<0.05)^{\mathrm{a}}\end{array}$ \\
\hline
\end{tabular}

Age

$\begin{array}{llll}<50 \text { years } & 39 & 20 & 19 \\ \geq 50 \text { years } & 85 & 48 & 37\end{array}$

0.698

Gender

$\begin{array}{llll}\text { Male } & 92 & 50 & 42 \\ \text { Female } & 32 & 18 & 14\end{array}$

Tumor size

(cm)

$<5 \mathrm{~cm}$

88

40

48

$\geq 5 \mathrm{~cm}$

36

28

8

Tumor

number

Solitary

Multiple

106

58

10

48

8

Edmondson

I+II
III+IV
TNM stage
I+II

I+II
III+IV

77

$$
36
$$

47

32

$0.021^{\mathrm{a}}$

$0.001^{\mathrm{a}}$

0.852

4

(nerase activity was measured using the dual-luciferase reporter assay system (Promega). Firefly luciferase activity was normalized to the Renilla luciferase activity. Results were obtained from three independent experiments performed in triplicate.

In vivo experiments. Four-to-six-week-old female BALB/c nude mice (Centre of Laboratory Animals, The Medical College of Xi'an Jiaotong University, Xi'an, China) were used to establish the nude mouse xenograft model. SMMC-7721 $\left(5 \times 10^{6}\right)$ cells that were transfected with anti-miR-155 or antimiR-NC vectors were mixed in $150 \mu 1$ of Matrigel and were inoculated subcutaneously into the flank of nude mice. The tumor volume for each mouse was determined by measuring two of its dimensions and then calculated as tumor volume $=$ length $\mathrm{x}$ width $\mathrm{x}$ width $/ 2$. After 3 weeks, the mice were sacrificed by cervical dislocation under anesthesia with ether and the xenograft tumor tissue was explanted for examination. Animal protocols were approved by the Institutional Animal Care and Use Committee of Xi'an Jiaotong University.

0.947

Statistical analysis. Data are presented as the mean \pm SD from at least three independent replicates. SPSS software, 16.0 (SPSS, Inc, Chicago, IL, USA) was used to conduct the analysis, and a two-tailed Student's t-test was employed to analyze the differences between two groups. Pearson's correlation analysis was used to analyze the correlation between two indices. Survival curves were plotted by the Kaplan-Meier method and compared by the log-rank test. Differences were considered statistically significant at $\mathrm{P}<0.05$.

\section{Results}

0.826

infiltration

\begin{tabular}{lrrrr} 
Present & 81 & 45 & 36 & \\
Absent & 43 & 23 & 20 & \\
Venous & & & & 0.668 \\
infiltration & & & & \\
$\quad$ Present & 15 & 9 & 6 & 0.569 \\
Absent & 109 & 59 & 50 & \\
AFP & & & & \\
$<400 \mathrm{ng} / \mathrm{ml}$ & 41 & 21 & 20 & \\
$\geq 400 \mathrm{ng} / \mathrm{ml}$ & 83 & 47 & 36 & \\
HBsAg & & & & \\
Positive & 115 & 63 & 52 & \\
Negative & 9 & 5 & 4 & \\
\hline
\end{tabular}

or mt ARID2-3'UTR, respectively. Then, SMMC-7721 cells $\left(1 \times 10^{5}\right)$ were seeded into 24 -well plates, and were cultured in OptimMEM reduced serum media (Life Technologies) as per the recommended conditions, and were cotransfected with $200 \mathrm{ng}$ of each luciferase reporter construct (the wt or mt 3'-UTR of ARID2 mRNA) and miR-155 expression vector, miR-155 inhibitor, control vector or negative control (50 nM) using FuGENE (Promega). After $48 \mathrm{~h}$, the cells were harvested
miR-155 is upregulated in HCC tissues and cells. To evaluate the potential role of miR-155 in HCC, we first quantified miR-155 in 124 pairs of HCC tissues and matched adjacent non-tumor tissues using qRT-PCR methods. As shown in Fig. 1A, miR-155 was significantly increased in the HCC tissues compared with adjacent non-tumor tissues. Furthermore, we determined the expression level of miR-155 in HCC cell lines and the normal hepatocyte cell line LO2. Similarly, miR-155 was significantly upregulated in all HCC cell lines compared with LO2 cells $(\mathrm{P}<0.05$, Fig. 1B). These results suggest that miR-155 expression is upregulated in HCC and may contribute to the development of HCC.

Clinical significance of elevated miR-155 expression in HCC specimens. To investigate the clinical significance of miR-155 in HCC, the patients were classified into high and low miR-155 expression subgroups with the median level of miR-155 as the cut-off. As shown in Table I, higher levels of miR-155 were significantly correlated with a large tumor size $(\geq 5 \mathrm{~cm}$; $\mathrm{P}=0.001$ ), high histological grade (Edmondson-Steiner grade III + IV; $\mathrm{P}=0.021$ ) and advanced tumor stage (TNM stage III + IV; $\mathrm{P}=0.020$ ) (Table I). To further evaluate the significant contribution of miR-155 expression in the prognosis of patients with HCC, we constructed Kaplan-Meier survival curves using the overall 5-year patient survival data to analyze cases with high and low miR-155 expression. As shown in Fig. 2, patients with high miR-155 expression exhibited worse 
A

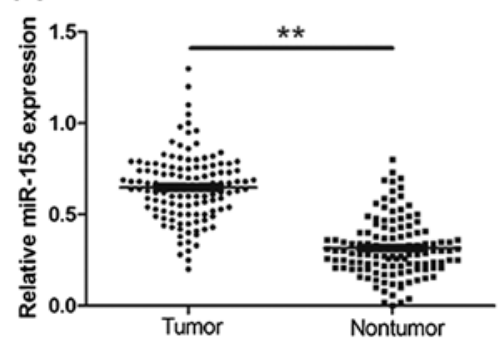

B

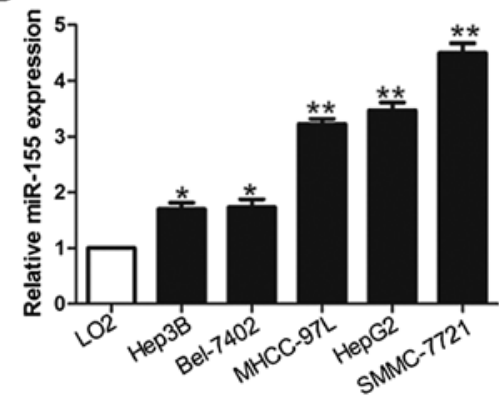

Figure 1. miR-155 is highly expressed in HCC tissues and cell lines. (A) Relative miR-155 expression levels in HCC tissues and matched adjacent non-tumor tissues were determined by qRT-PCR $(n=124)$. (B) The expression of miR-155 in HCC cell lines was significantly increased compared to that in the LO2 cells. U6 snRNA was used as internal control. ${ }^{*} \mathrm{P}<0.05,{ }^{* *} \mathrm{P}<0.01$.
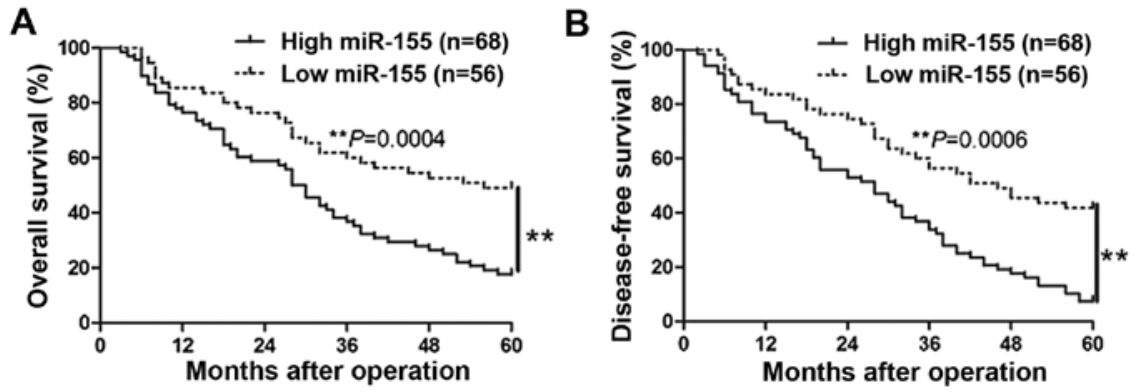

Figure 2. The prognostic value of miR-155 for HCC patients assessed by Kaplan-Meier analysis. HCC patients with high expression of miR-155 had worse (A) overall survival (OS) and (B) disease-free survival (DFS). ${ }^{* *} \mathrm{P}<0.01$.
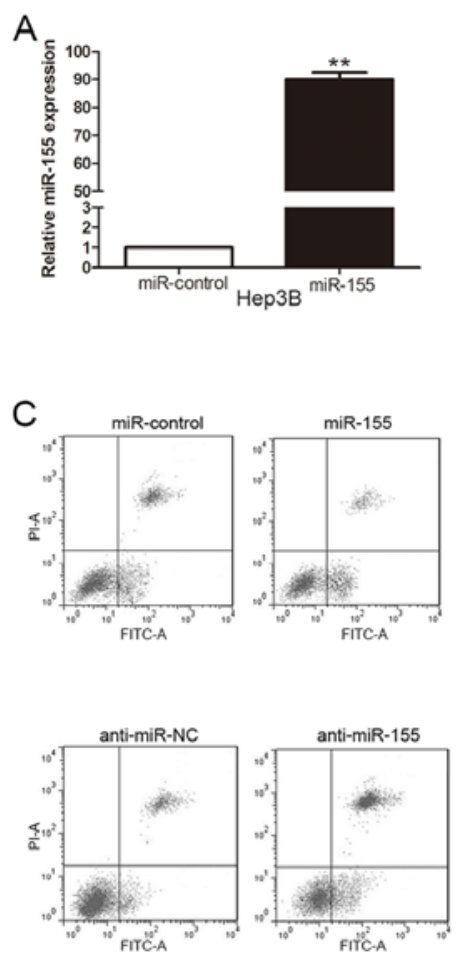

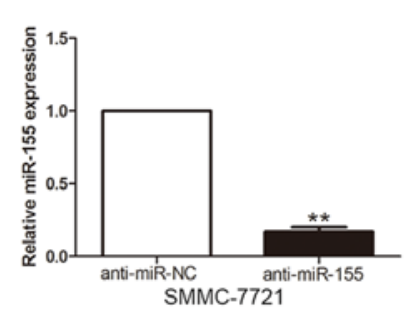

\section{B}
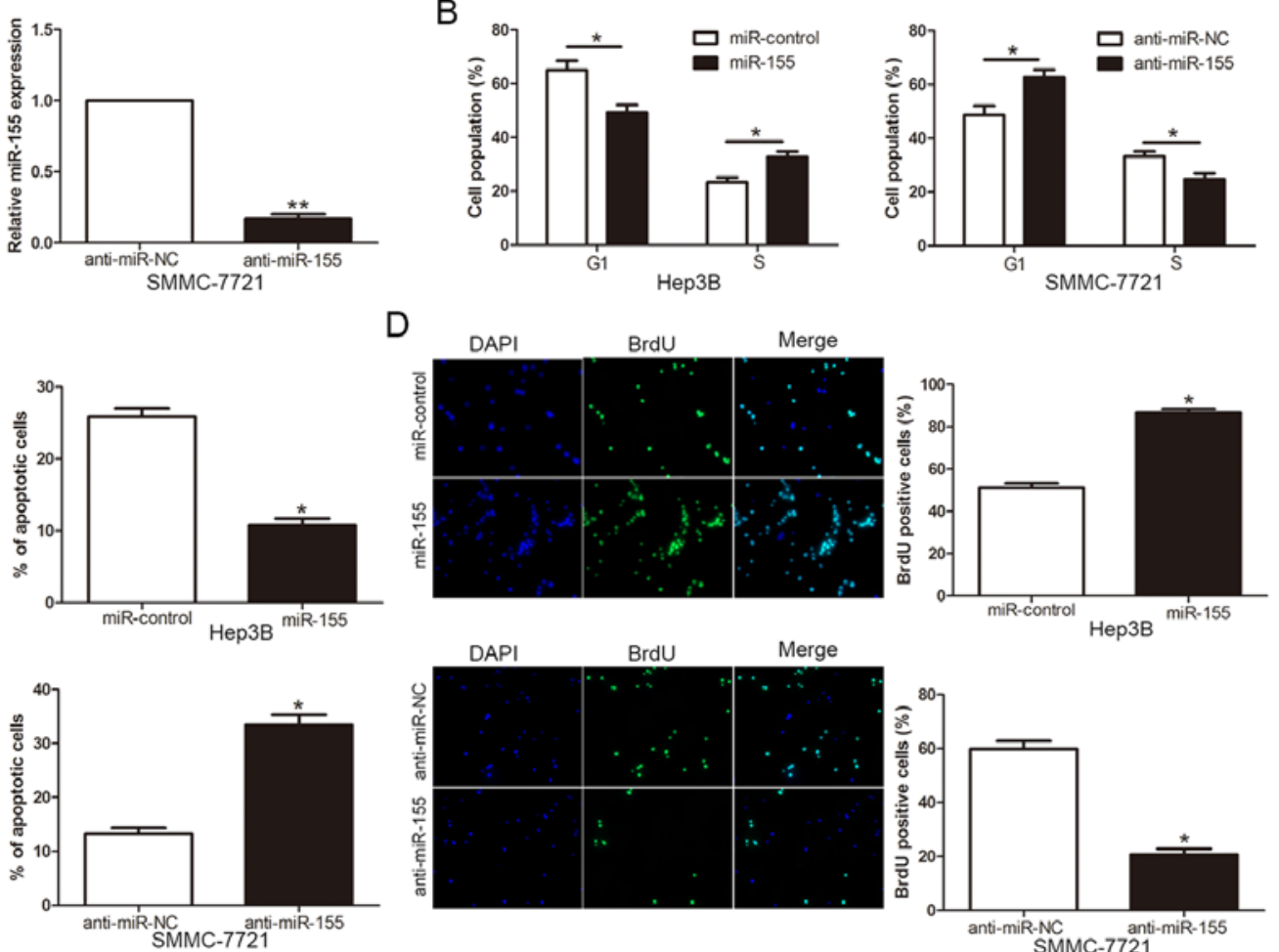

Figure 3. miR-155 promotes HCC cell cycle transition, cell proliferation and apoptosis resistance. (A) Hep3B and SMMC-7721 cells that were transfected with corresponding miRNA vectors were subjected to qRT-PCR for miR-155. (B) As assessed by flow cytometry, overexpression of miR-155 promoted cell cycle transition from G1 to S-phase in Hep3B cells and knockdown of miR-155 induced G1 phase arrest in SMMC-7721 cells. (C) Quantification of the apoptotic cell population by flow cytometry. Overexpression of miR-155 inhibited apoptosis in Hep3B cells and knockdown of miR-155 increased the percentage of apoptotic SMMC-7721 cells compared with the control cells. (D) Cell proliferation as measured by BrdU incorporation assays was increased by overexpression of miR-155 in Hep3B cells and inhibited by knockdown of miR-155 in SMMC-7721 cells. Representative micrographs (left) and quantification (right) of BrdU incorporating-cells of indicated cells. Experiments were repeated at least 3 times with similar results, and error bars represent mean $\pm \mathrm{SD}$. ${ }^{*} \mathrm{P}<0.05$, ${ }^{*} \mathrm{P}<0.01$. 
A

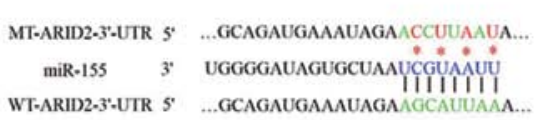

B

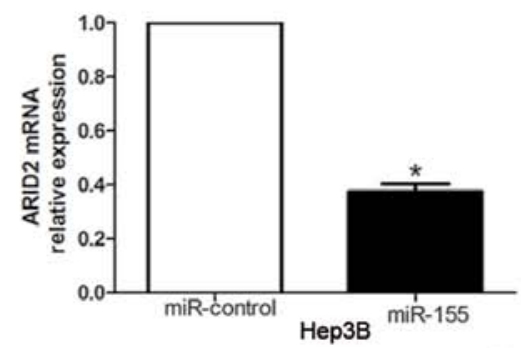

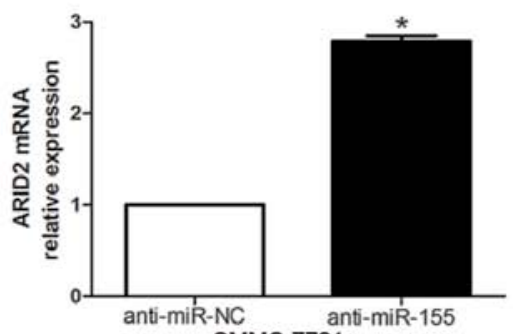

SMMC-7721
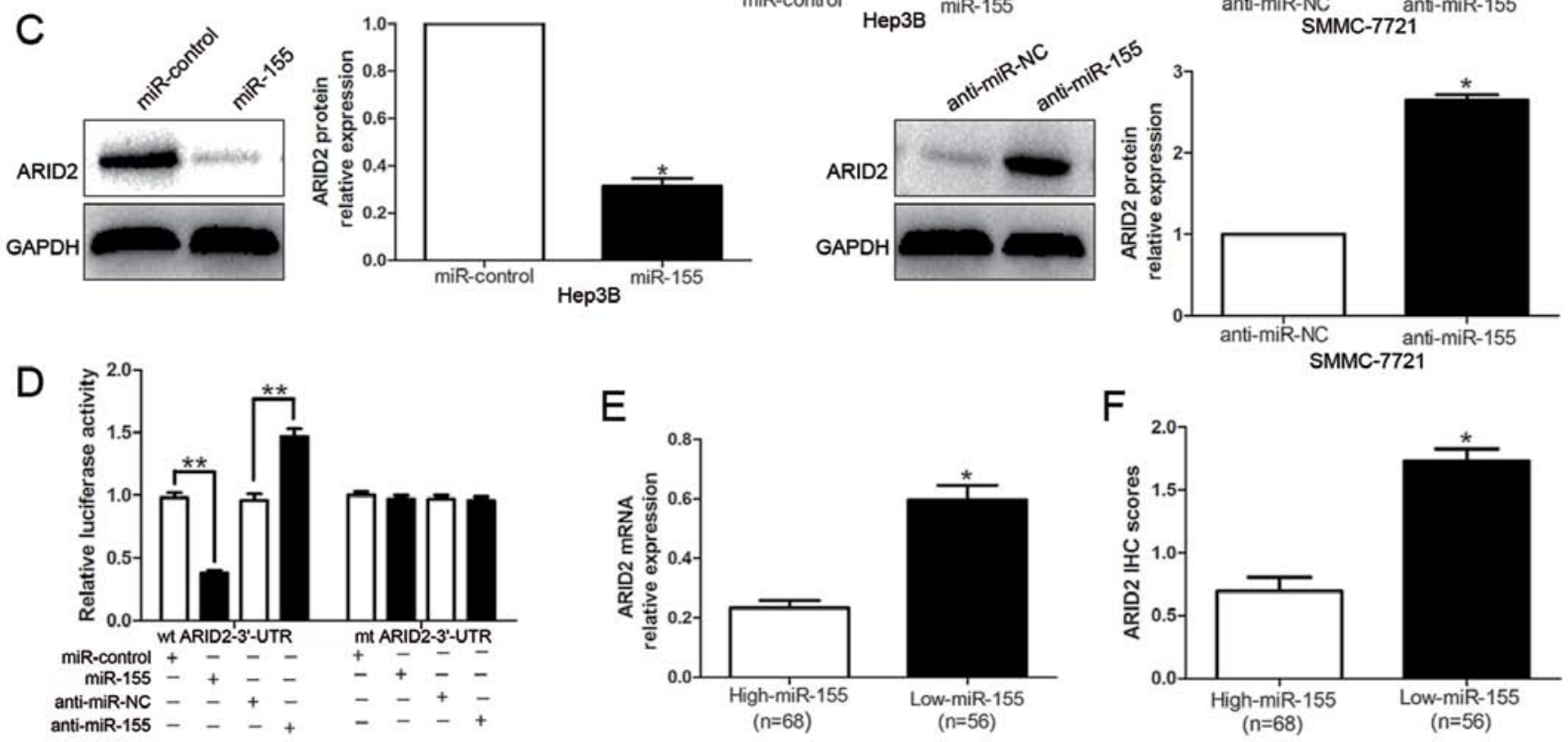

E

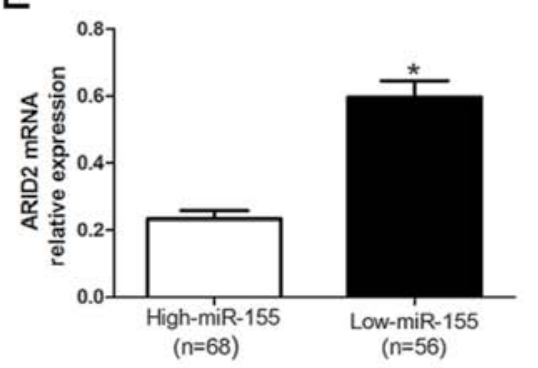

$\mathrm{F}$
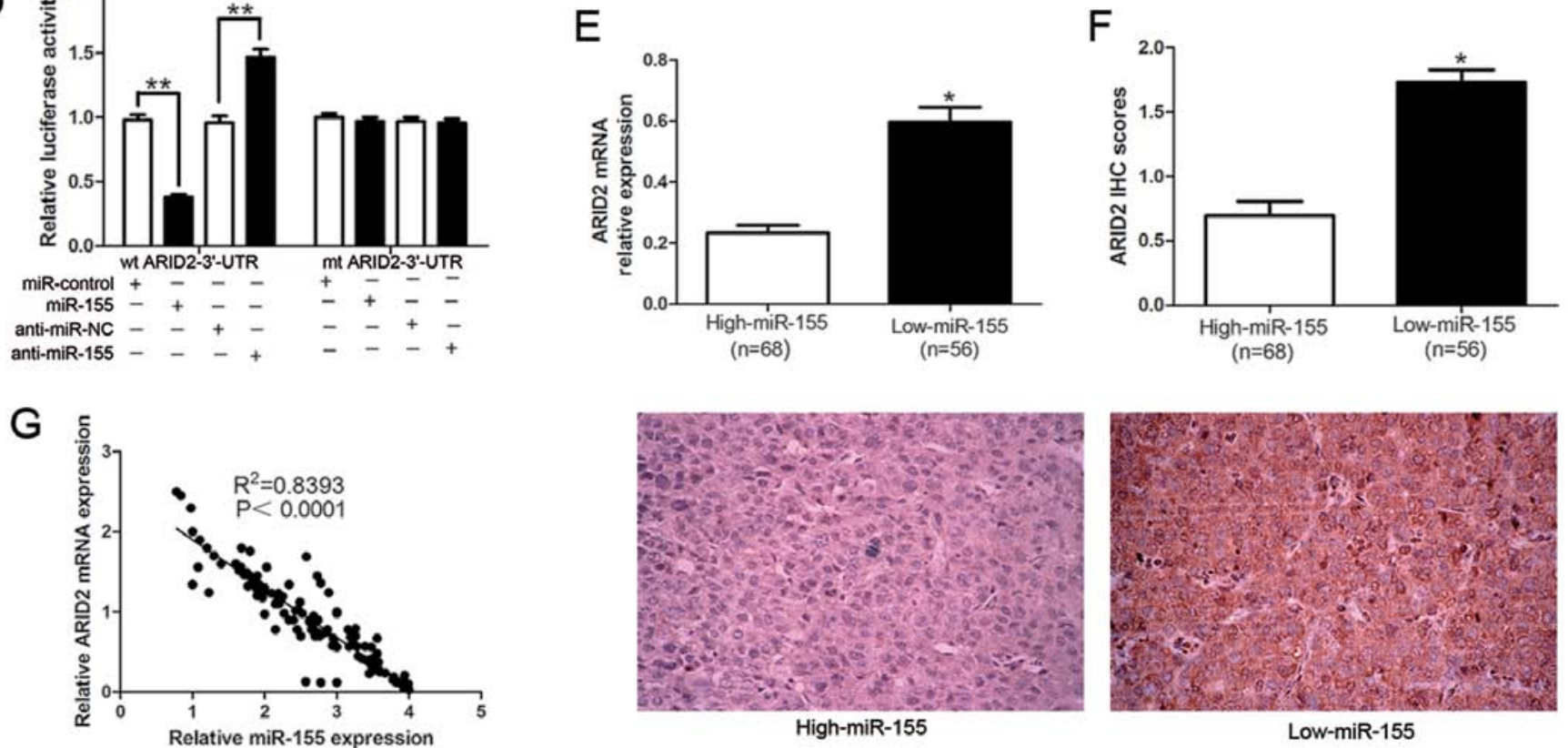

Figure 4. ARID2 is a direct target of miR-155 in HCC cells. (A) TargetScan demonstrated that 3'-UTR of ARID2 contained the highly conserved putative miR-155 binding sites. (B) qRT-PCR analysis of ARID2 mRNA expression in Hep3B cells with miR-155 or control (miR-control) transfection and SMMC-7721 cells with anti-miR-155 or anti-miR-NC (negative control) vector transfection. (C) Overexpression of miR-155 reduced the expression of ARID2 protein in Hep3B cells and knockdown of miR-155 increases the level of ARID2 protein in SMMC-7721 cells. (D) miR-155 significantly suppresses the luciferase activity that carried wild-type (wt) but not mutant (mt) 3'-UTR of ARID2. Anti-miR-155 led to a notable increase in the luciferase activity of wt 3'-UTR of ARID2. (E) The expression of ARID2 mRNA in miR-155 high-expressing tumors was significantly lower than that in miR-155 low-expressing tumors. (F) A significant inverse correlation between miR-155 and ARID2 protein expression was observed in HCC tissues. Representative immunohistochemical staining showed a weak staining of ARID2 in miR-155 high-expressing HCC tissue and strong staining of ARID2 in the miR-155 low-expressing tumor. (G) A statistically significant inverse correlation was shown between miR-155 and ARID2 mRNA levels in HCC specimens by Spearman's correlation analysis. Experiments were repeated at least 3 times with similar results. ${ }^{*} \mathrm{P}<0.05,{ }^{* *} \mathrm{P}<0.01$.

overall survival $(\mathrm{P}=0.0004)$ and disease-free survival $(\mathrm{P}=0.0006)$. These data indicate that miR-155 could serve as a valuable indicator for predicting the prognosis of HCC.

miR-155 promotes cell cycle progression, cell proliferation and inhibits apoptosis in HCC cells. To investigate the biological function of miR-155 in the development and progression of HCC, we transduced a miR-155 expression vector or a miR-155 inhibitor (anti-miR-155) into Hep3B and SMMC-7721 cells, respectively. As measured by qRT-PCR, miR-155 expression vector significantly increased the level of miR-155 in Hep3B cells, while the anti-miR-155 vector significantly reduced the expression of miR-155 in SMMC-7721 cells ( $\mathrm{P}<0.01$, Fig. 3A). As determined by flow cytometric analysis, the upregulation of miR-155 promoted cell cycle transition from $\mathrm{G} 1$ to $\mathrm{S}$ phase $(\mathrm{P}<0.05$, Fig. 3B) and apoptosis resistance $(\mathrm{P}<0.05$, Fig. $3 \mathrm{C})$ in Hep3B cells. Furthermore, the overexpression of miR-155 significantly increased cell proliferation examined with incorporation assay in Hep3B cells $(\mathrm{P}<0.05$, Fig. 3D). By contrast, the downregulation of $\mathrm{miR}-155$ resulted in $\mathrm{G} 1$ arrest, apoptosis promotion and proliferation reduction in SMMC-7721 cells $(\mathrm{P}<0.05$, respectively, Fig. 3B-D). These results demonstrated 

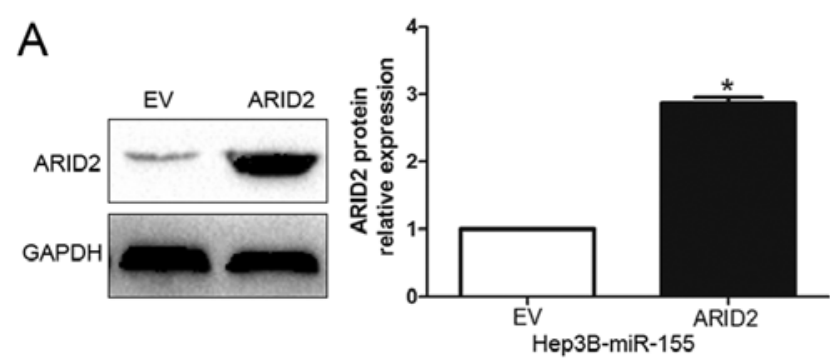

\section{B}
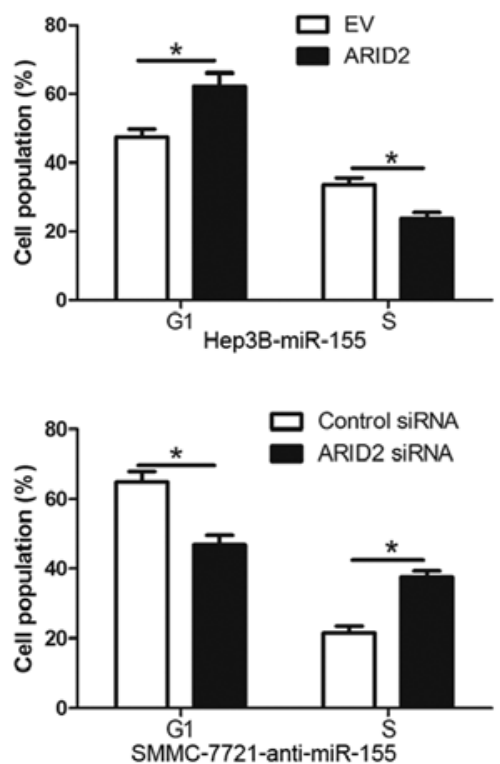

C
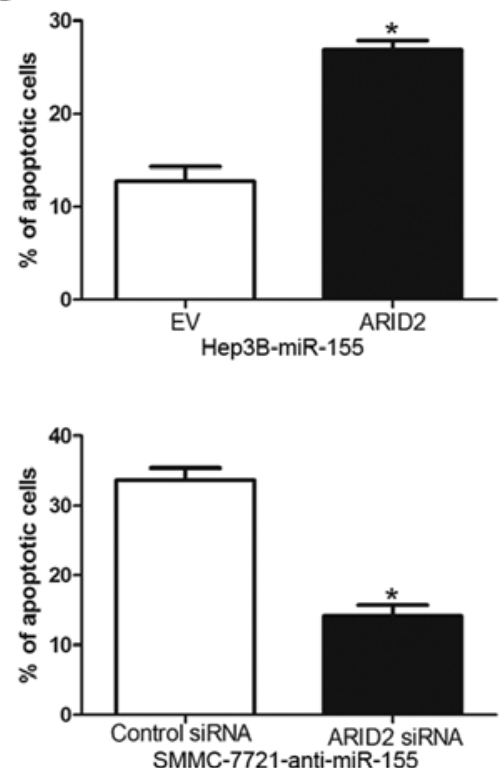

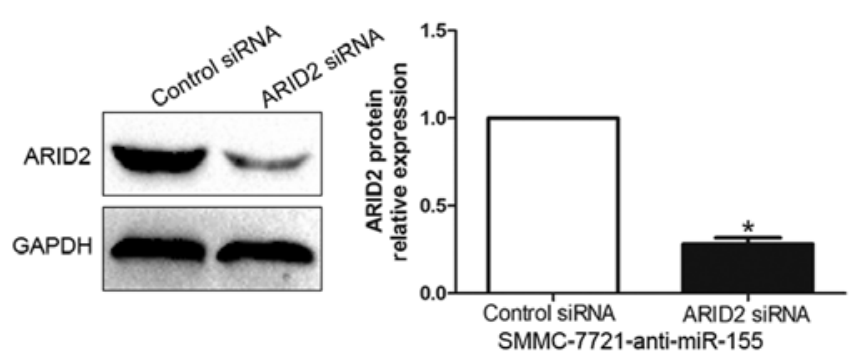

D
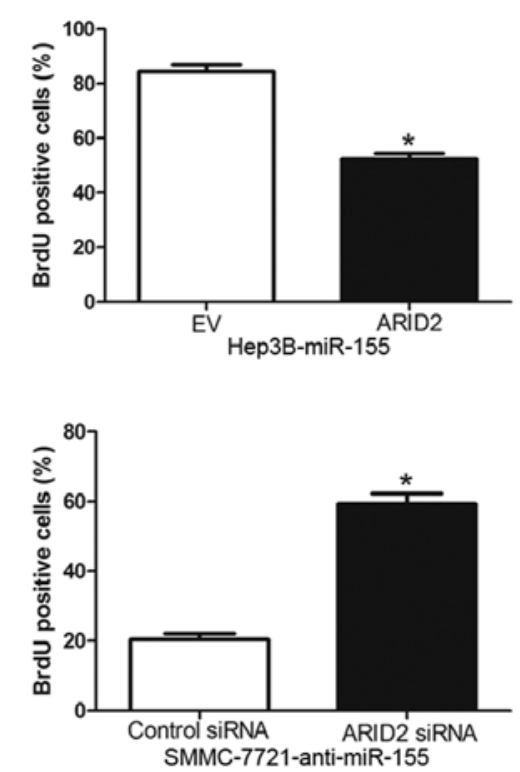

Figure 5. Alterations of ARID2 partially abolish miR-155-mediated HCC cell cycle progression, proliferation and apoptosis. (A) miR-155-overexpressing Hep3B that were transfected with EV or ARID2 expression plasmid and miR-155-suppressive SMMC-7721 cells that were transfected with control siRNA or ARID2 siRNA were subjected to western blot analysis for ARID2. (B) G1 phase arrest was induced after ARID2 overexpression in miR-155-overexpressing Hep3B cells. The cell cycle transition from G1 to S-phase was increased by ARID2 knockdown in miR-155-suppressive SMMC-7721 cells. The correlations between miR-155 effects and ARID2 overexpression or knockdown are shown in (C) apoptosis and (D) cell proliferation. ARID2 overexpression induced effects that were opposite to those stimulated by miR-155 overexpression in Hep3B cells. ARID2 knockdown abrogated the effects of miR-155 knockdown on SMMC-7721 cells. $\mathrm{n}$, three independent experiments. ${ }^{*} \mathrm{P}<0.05,{ }^{* *} \mathrm{P}<0.01$.

that miR-155 regulates the cell cycle progression, apoptosis and proliferation of HCC cells.

ARID2 is a direct target of miR-155 in HCC. To explore the mechanism of miR-155 regulation in HCC, we used publicly available databases TargetScan 6.2 and miRanda to search predicted genes. Among them, the binding sites for miR-155 on the 3'-UTR of ARID2 were conserved among species (Fig. 4A). To verify the regulation role of miR-155 on ARID2, qRT-PCR and western blotting were performed to detect the effect of miR-155 on ARID2 mRNA and protein levels. Ectopic expression of miR-155 markedly decreased, while inhibition of miR-155 increased the ARID2 mRNA $(\mathrm{P}<0.05$, Fig. 4B) and protein $(\mathrm{P}<0.05$, Fig. $4 \mathrm{C})$. In addition, the overexpression of miR-155 prominently inhibited the luciferase activity of ARID2 containing a wild-type (wt) 3'-UTR but did not suppress the activity of ARID2 with a mutant (mt) 3'-UTR $(\mathrm{P}<0.01$, Fig. 4D). Suppression of miR-155 by anti-miR-155 increased the luciferase activity of wt ARID2 3'-UTR $(\mathrm{P}<0.01$, Fig. 4D). However, with the mt ARID2 3'-UTR constructs, there was no relative increase in activity. Moreover, the expression levels of ARID2 mRNA and protein in the high miR-155 expression tumors were significantly lower than those in the low miR-155 expression tumors $(\mathrm{P}<0.05$, respectively, Fig. $4 \mathrm{E}$ and F). Notably, the expression level of miR-155 was inversely correlated with the level of ARID2 mRNA in HCC tissues $\left(\mathrm{R}^{2}=0.8393, \mathrm{P}<0.0001\right.$, Fig. $\left.4 \mathrm{G}\right)$. On the basis of these data, we conclude that ARID2 is a direct target gene for miR-155 and that miR-155 downregulates ARID2 expression.

Altering expression of ARID2 influences the effect of $m i R-155$ on HCC cells. To confirm that ARID2 is a functional target of miR-155, we restored ARID2 expression in Hep3B-miR-155 cells by transfecting ARID2 expression plasmid $(\mathrm{P}<0.05$, Fig. 5A). Functionally, restoration of ARID2 expression in Hep3B-miR-155 cells partially abrogated the effect of exogenous miR-155, resulting in significant increase of apoptosis $(\mathrm{P}<0.01$, Fig. 5C) and obvious decrease of cell cycle progression and cell proliferation ( $\mathrm{P}<0.05$, respectively, Fig. 5B and $\mathrm{D})$. Similarly, silencing of ARID2 in SMMC-7721-anti-miR-155 cells partially abolished the effect of anti-miR-155 on cell cycle, apoptosis and proliferation ( $\mathrm{P}<0.05$, respectively, Fig. 5). These results demonstrate that ARID2 is a downstream mediator for the function of miR-155 in HCC. 
A

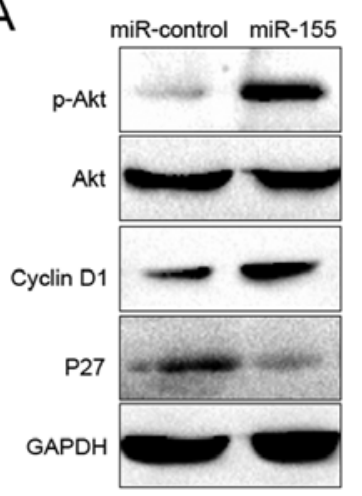

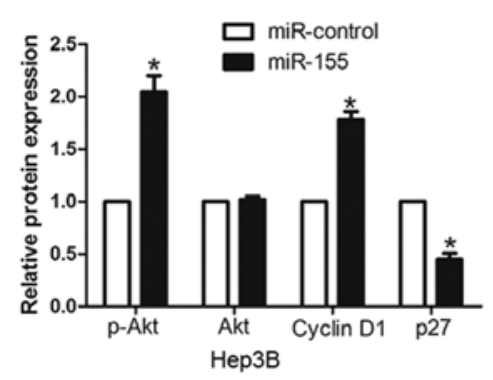

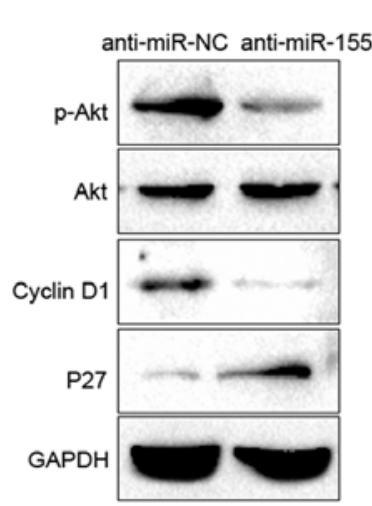

B
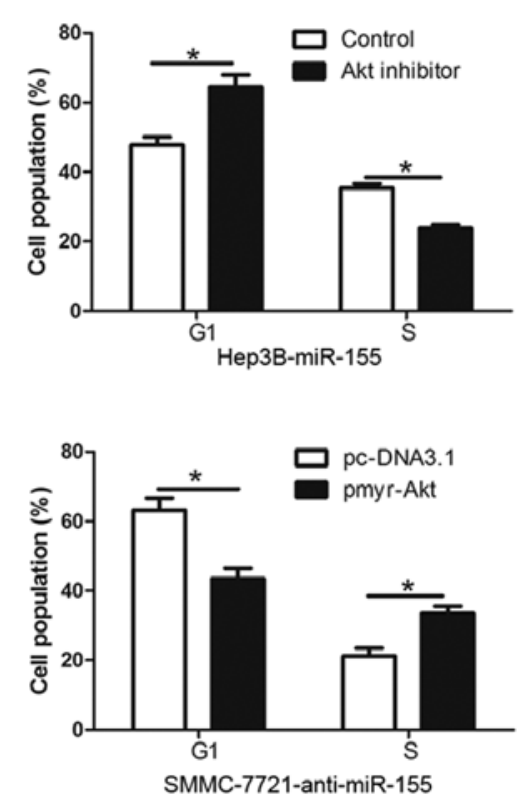

C
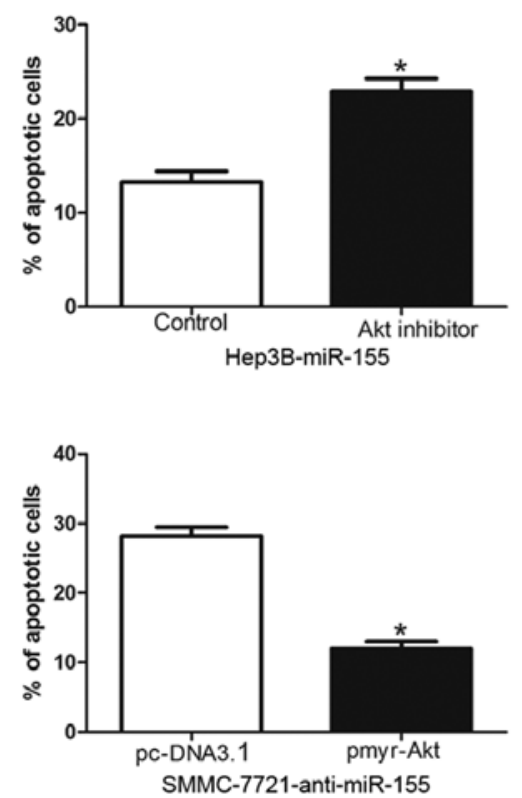

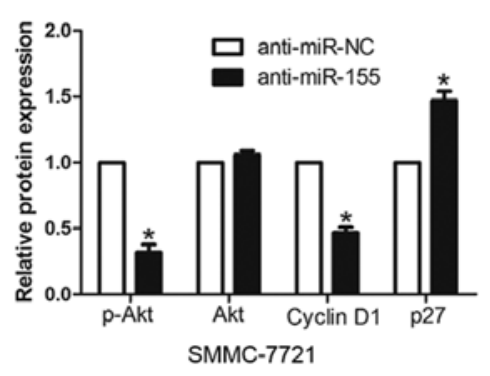

D
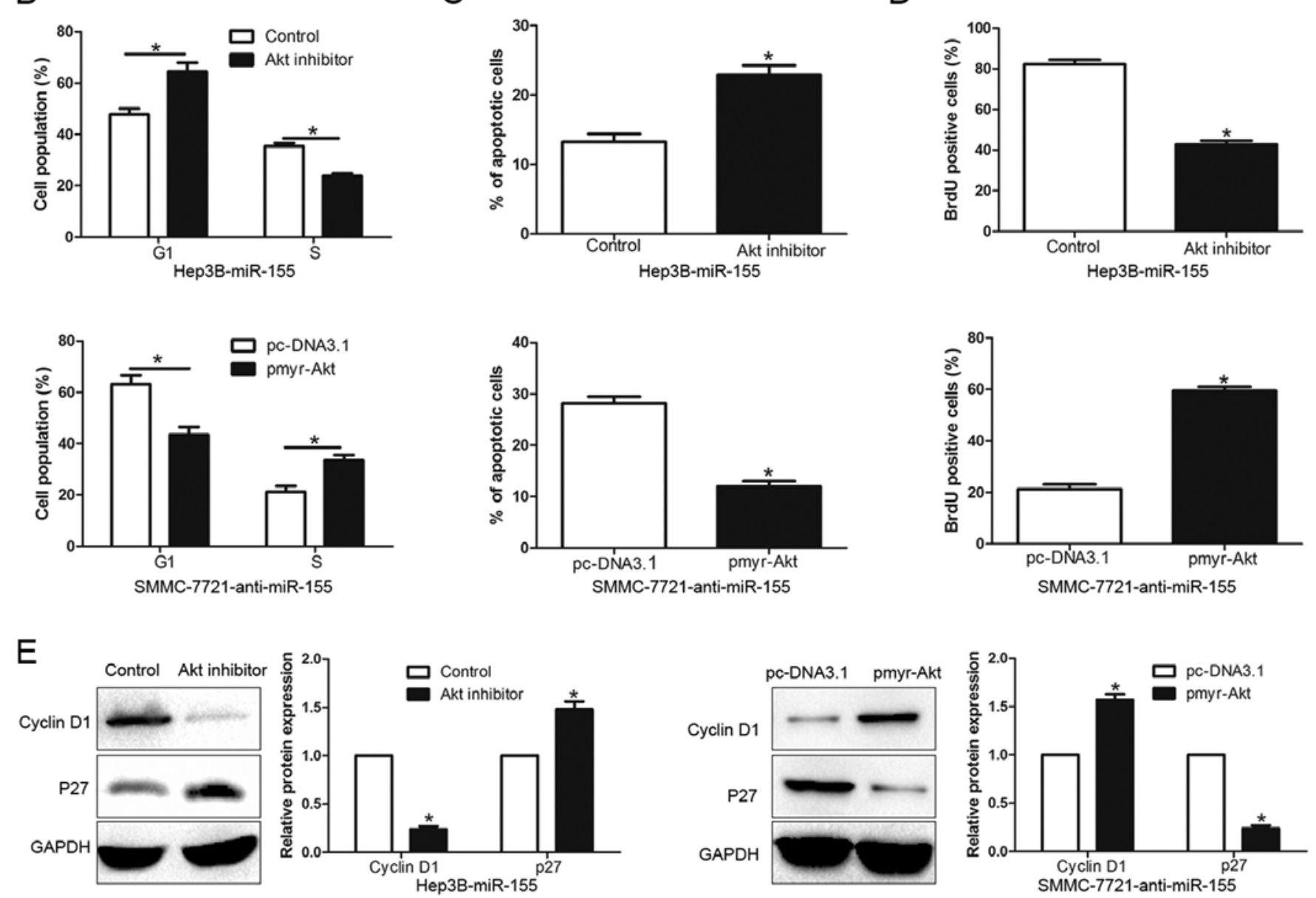

Figure 6. Activated Akt phosphorylation signaling is essential for miR-155-promoted HCC cell cycle progression, proliferation and miR-155-inhibited apoptosis. (A) Western blot analysis of p-Akt, total Akt, Cyclin D1 and p27 protein levels in indicated cells. Quantification of cell cycle (B), apoptosis (C) and cell proliferation (D) in indicated HCC cells treated with Akt inhibitor $(0.5 \mu \mathrm{M})$ or transfected with dominant-active Akt plasmid. (E) The protein expression level of Cyclin D1 and p27 by western blot analysis in indicated HCC cells treated with Akt inhibitor $(0.5 \mu \mathrm{M})$ or transfected with dominant-active Akt plasmid. Experiments were repeated at least 3 times with similar results, and error bars represent mean \pm SD. ${ }^{*} \mathrm{P}<0.05$.

Akt phosphorylation is essential for the biological function of miR-155 in HCC. Previous studies demonstrated that activation of Akt signaling played an important role in HCC cell cycle progression, apoptosis and proliferation (20-22), so we further investigated the underlying molecular mechanisms of the miR-155-mediated promotion of HCC biological effects. As shown in Fig. 6A, ectopic expression of miR-155 significantly increased, while miR-155 inhibition decreased, the Akt phosphorylation in HCC cells $(\mathrm{P}<0.05)$. Consistently, the expression of Cyclin D1 and p27, which are the downstream effectors of Akt signaling and the key regulators of cell cycle progression and proliferation in HCC, were also altered in the up- or down-expression of miR-155 HCC cells $(\mathrm{P}<0.05)$. Furthermore, to confirm that Akt phosphorylation contributed to the biological function of miR-155-mediated in HCC cells, we used Akt inhibitor or pmyr-Akt (dominantactive Akt) plasmid to affect Akt activation. Inactivation of Akt phosphorylation by Akt inhibitor significantly decreased 
A

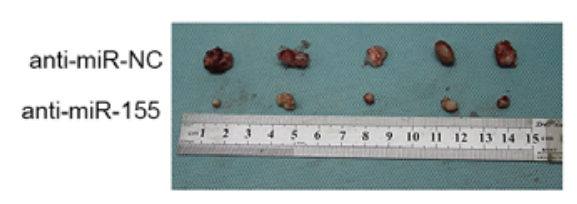

B

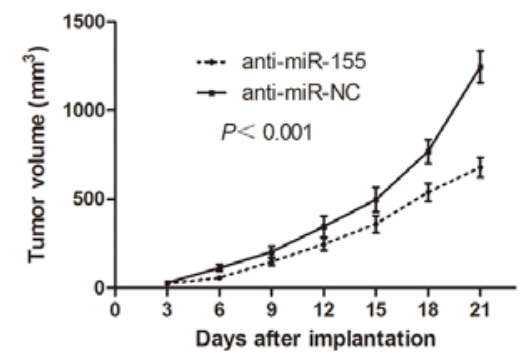

D

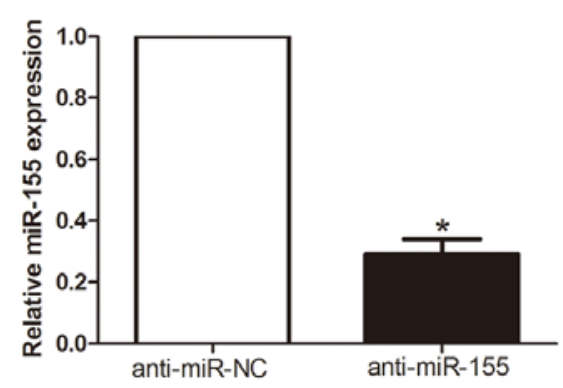

E

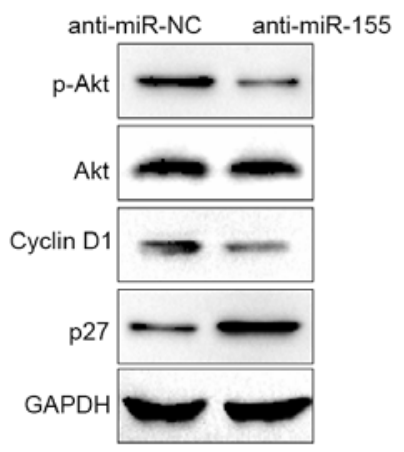

C

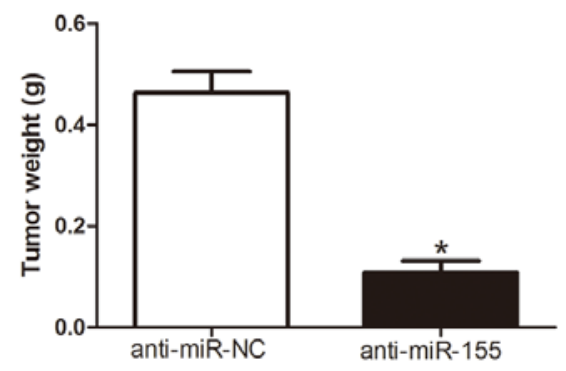

Figure 7. miR-155 promotes HCC growth by activating Akt signaling in vivo. (A) Representative images of HCC xenografts from both SMMC-7721 antimiR-NC (upper panel) and SMMC-7721-anti-miR-155 cells (lower panel). Tumor growth curve (B) and weight (C) revealed that miR-155 knockdown significantly inhibited tumor growth in vivo. (D) Expression of miR-155 in miR-155 inhibitor-treated tumors from the subcutaneous model. (E) Expression of p-Akt, Akt, Cyclin D1, p27 were assessed in the subcutaneous model by western blotting. ${ }^{*} \mathrm{P}<0.05,{ }^{* *} \mathrm{P}<0.01$.

cell cycle progression $(\mathrm{P}<0.05$, Fig. 6B) and proliferation $(\mathrm{P}<0.05$, Fig. 6D) and induced apoptosis $(\mathrm{P}<0.05$, Fig. 6C) in Hep3B-miR-155 cells. In addition, Akt overexpression of phosphorylation increased cell cycle progression, cell proliferation and inhibited apoptosis $(\mathrm{P}<0.05$, respectively, Fig. 6B-D) in SMMC-7721-anti-miR-155 cells. Accordingly, the expression of Cyclin D1 and p27 was also significantly altered $(\mathrm{P}<0.05$, Fig. $6 \mathrm{E})$. Taken together, our results demonstrate that Akt phosphorylation exerts an important role in miR-155-mediated HCC cell cycle progression, apoptosis and proliferation.

miR-155 promotes HCC cell cycle progression, proliferation and inhibits apoptosis in vivo. To further confirm our results in vivo, SMMC-7721 cells were transfected with anti-miR-155 or anti-miR-NC vector and implanted subcutaneously into nude mice (Fig. 7A). Notably, the tumor growth curve revealed that knockdown of miR-155 significantly retarded the tumor growth of human HCC in the subcutaneous nude mouse model ( $\mathrm{P}<0.001$, Fig. 7B). As shown in Fig. 7C, in the miR-155 knockdown group, the tumor weight was smaller than that in the control group. Furthermore, the downregulation of miR-155 in the tumor formation in the subcutaneous model $(\mathrm{P}<0.05$, Fig. 7D), inhibited the activation of Akt signaling $(\mathrm{P}<0.05$, Fig. 7E). Taken together, these results demonstrated that miR-155 regulates the cell cycle progression, apoptosis and cell proliferation of HCC cells and contributed to the development and progression of HCC.

\section{Discussion}

miRNAs are known to be regulators of many genes at the post-transcript level, and their aberrant expression is related to cancer initiation, development and prognosis (23). However, the molecular mechanisms by which miRNAs modulate the biological function of cancer cells are still largely unknown. In previous studies, miR-155 controls affinity-based selection by protecting c-MYC+ B cells from apoptosis (24). Lerner et al indicated that miR-155 was upregulated in blood, tissue and cell lines of head and neck squamous cell carcinoma and could serve as an independent prognosis predictor for patients and promoted the cell proliferation and migration (25). Moreover, Hou et al found that miR-155 promoted non-small cell lung carcinoma proliferation through inhibition of FoxO1 and the subsequent increase of ROS generation (26).

In this study, we initially investigated the expression of miR-155 in 124 paired samples of HCC and non-tumor tissues. Our data showed that the expression of miR-155 was elevated in HCC tissues. Furthermore, miR-155 was upregulated in HCC cell lines as compared with a normal hepatic cell line. These results indicate that miR-155 may be an oncogene and may play a critical role in hepatocarcinogenesis. Importantly, its upregulation significantly correlated with the poorer prognosis of patients with HCC. In addition, elevated miR-155 expression was significantly correlated with malignant clinicopathological featurs of HCC, including large tumor size, high histological grade and TNM stage. miR-155 
has been found to promote proliferation of lung cancer by repressing FoxO1. We found that the downregulation of miR-155 inhibited HCC cell proliferation and induced G1 phase arrest and apoptosis in vitro. By contrast, the overexpression of miR-155 promoted proliferation and cell cycle transition to $\mathrm{S}$ phase and inhibited apoptosis. These results suggested that miR-155 is a novel tumor-promoting miRNA that plays a critical role in the regulation of tumor growth in HCC.

To further understand the underlying mechanisms by which miR-155 exerts its biological effects on HCC cells, it is necessary to identify its downstream functional targets. ARID2, a new tumor suppressor gene in HCC, was initially identified in the Polybromo-associated BRG1-associated factor (PBAF) complex (27), an SWI/SNF chromatin remodeling complex involved in ligand-dependent transcriptional activation by nuclear receptors (28). Downregulated expression of ARID2 has been observed in liver cancer cells (29). We identified ARID2 as a novel direct target of miR-155. Firstly, miR-155 negatively regulated ARID2 mRNA and protein in HCC cells. Secondly, overexpression of miR-155 decreased, while downregulation of miR-155 increased, the luciferase reporter activity of ARID2 wt 3'-UTR, but not mt 3'-UTR. Thirdly, miR-155 was inversely correlated with the levels of both ARID2 mRNA and protein in HCC tissues. Finally, the effects of miR-155 alteration on cell cycle progression, apoptosis and proliferation were also abolished by ARID2 modulation. Collectively, our results support that miR-155 exerts its biological function on HCC through targeting ARID2.

It has been well-established that Akt phosphorylation could promote cell cycle progression and proliferation through upregulation of Cyclin D1 and downregulation of p27 (22). As miR-155 plays a critical role in cell fate, a better understanding of its regulatory axis might provide new opportunities for developing more effective therapeutic approaches to treat cancers. In addition, we discovered that miR-155 activated Akt phosphorylation through targeting ARID2. Consistently, we performed loss-experiment by Akt inhibitor, gain-experiment by dominant-active Akt plasmid to confirm that Akt phosphorylation plays an important role in the miR-155-mediated biological function. In addition, we discovered miR-155 activated Akt signaling through targeting ARID2 in vivo, suggesting miR-155 may represent as a potential therapeutic target for HCC treatment.

In conclusion, we find that miR-155 is upregulated in HCC tissues and cells, and its elevated expression is associated with poor prognostic features. In vitro and in vivo studies indicate that miR-155 promotes tumor growth by promoting HCC cell cycle progression, cell proliferation and inhibiting apoptosis. Mechanistically, we suggest that miR-155 exerts its biological function through Akt phosphorylation by targeting ARID2. Therefore, miR-155 has the potential to be a valuable diagnostic and prognostic biomarker for HCC.

\section{Acknowledgements}

This study was supported by the Science and Technology Research and Development Program of Shaanxi Province [2013k-12].

\section{References}

1. Forner A, Llovet JM and Bruix J: Hepatocellular carcinoma. Lancet 379: 1245-1255, 2012.

2. Tang ZY: Hepatocellular carcinoma surgery - review of the past and prospects for the 21st century. J Surg Oncol 91: 95-96, 2005.

3. El-Serag HB and Rudolph KL: Hepatocellular carcinoma: Epidemiology and molecular carcinogenesis. Gastroenterology 132: 2557-2576, 2007.

4. Lujambio A and Lowe SW: The microcosmos of cancer. Nature 482: 347-355, 2012.

5. Yates LA, Norbury CJ and Gilbert RJ: The long and short of microRNA. Cell 153: 516-519, 2013.

6. Corsini LR, Bronte G, Terrasi M, Amodeo V, Fanale D, Fiorentino E, Cicero G, Bazan V and Russo A: The role of microRNAs in cancer: Diagnostic and prognostic biomarkers and targets of therapies. Expert Opin Ther Targets 16 (Suppl 2): S103-S109, 2012.

7. Chang RM, Yang H, Fang F, Xu JF and Yang LY: MicroRNA331-3p promotes proliferation and metastasis of hepatocellular carcinoma by targeting $\mathrm{PH}$ domain and leucine-rich repeat protein phosphatase. Hepatology 60: 1251-1263, 2014.

8. Tu K, Liu Z, Yao B, Han S and Yang W: MicroRNA-519a promotes tumor growth by targeting PTEN/PI3K/AKT signaling in hepatocellular carcinoma. Int J Oncol 48: 965-974, 2016.

9. Dou C, Wang Y, Li C, Liu Z, Jia Y, Li Q, Yang W, Yao Y, Liu Q and Tu K: MicroRNA-212 suppresses tumor growth of human hepatocellular carcinoma by targeting FOXA1. Oncotarget 6 : 13216-13228, 2015.

10. Chai ZT, Kong J, Zhu XD, Zhang YY, Lu L, Zhou JM, Wang LR, Zhang KZ, Zhang QB, Ao JY, et al: MicroRNA-26a inhibits angiogenesis by down-regulating VEGFA through the PIK3C $2 \alpha /$ Akt/HIF-1 $\alpha$ pathway in hepatocellular carcinoma. PLoS One 8: e77957, 2013.

11. Sun S, Sun P, Wang Cand Sun T: Downregulation of microRNA-155 accelerates cell growth and invasion by targeting c-myc in human gastric carcinoma cells. Oncol Rep 32: 951-956, 2014.

12. Li T, Yang J, Lv X, Liu K, Gao C, Xing Y and Xi T: miR-155 regulates the proliferation and cell cycle of colorectal carcinoma cells by targeting E2F2. Biotechnol Lett 36: 1743-1752, 2014.

13. Wang F, Zhou J, Zhang Y, Wang Y, Cheng L, Bai Y and Ma H: The value of microRNA-155 as a prognostic factor for survival in non-small cell lung cancer: A meta-analysis. PLoS One 10: e0136889, 2015.

14. Bertoli G,Cava $C$ and Castiglioni I: MicroRNAs: New biomarkers for diagnosis, prognosis, therapy prediction and therapeutic tools for breast cancer. Theranostics 5: 1122-1143, 2015

15. Zhang X, Zhang Y, Liu X, Fang A, Wang J, Yang Y, Wang L, Du L and Wang C: Direct quantitative detection for cell-free miR-155 in urine: A potential role in diagnosis and prognosis for non-muscle invasive bladder cancer. Oncotarget 7: 3255-3266, 2016.

16. Slezak-Prochazka I, Kluiver J, de Jong D, Smigielska-Czepiel K, Kortman G, Winkle M, Rutgers B, Koerts J, Visser L, Diepstra A, et al: Inhibition of the miR-155 target NIAM phenocopies the growth promoting effect of miR-155 in B-cell lymphoma. Oncotarget 7: 2391-2400, 2016.

17. Baba O, Hasegawa S, Nagai H, Uchida F, Yamatoji M, Kanno NI, Yamagata K, Sakai S, Yanagawa T and Bukawa H: MicroRNA$155-5 \mathrm{p}$ is associated with oral squamous cell carcinoma metastasis and poor prognosis. J Oral Pathol Med: Aug 26, 2015 (Epub ahead of print). doi: 10.1111/jop.12351.

18. Han S, Yang S, Cai Z, Pan D, Li Z, Huang Z, Zhang P, Zhu H, Lei $\mathrm{L}$ and Wang W: Anti-Warburg effect of rosmarinic acid via miR-155 in gastric cancer cells. Drug Des Devel Ther 9: 2695-2703, 2015 .

19. Shen R, Wang Y, Wang CX, Yin M, Liu HL, Chen JP, Han JQ and Wang WB: MiRNA-155 mediates TAM resistance by modulating SOCS6-STAT3 signalling pathway in breast cancer. Am J Transl Res 7: 2115-2126, 2015.

20. Hou YQ, Yao Y, Bao YL, Song ZB, Yang C, Gao XL, Zhang WJ, Sun LG, Yu CL, Huang YX, et al: Juglanthraquinone C induces intracellular ROS increase and apoptosis by activating the Akt/ Foxo signal pathway in HCC cells. Oxid Med Cell Longev 2016: $4941623,2016$.

21. Ewald F, Nörz D, Grottke A, Bach J, Herzberger C, Hofmann BT, Nashan B and Jücker M: Vertical targeting of AKT and mTOR as well as dual targeting of AKT and MEK signaling is synergistic in hepatocellular carcinoma. J Cancer 6: 1195-1205, 2015. 
22. Liu X, Liao W, Yuan Q, Ou Y and Huang J: TTK activates Akt and promotes proliferation and migration of hepatocellular carcinoma cells. Oncotarget 6: 34309-34320, 2015.

23. Murakami Y, Yasuda T, Saigo K, Urashima T, Toyoda H, Okanoue $\mathrm{T}$ and Shimotohno K: Comprehensive analysis of microRNA expression patterns in hepatocellular carcinoma and non-tumorous tissues. Oncogene 25: 2537-2545, 2006.

24. Nakagawa R, Leyland R, Meyer-Hermann M, Lu D, Turner M, Arbore G, Phan TG, Brink R and Vigorito E: MicroRNA-155 controls affinity-based selection by protecting c-MYC ${ }^{+} \mathrm{B}$ cells from apoptosis. J Clin Invest 126: 377-388, 2016.

25. Lerner C, Wemmert S, Bochen F, Kulas P, Linxweiler M, Hasenfus A, Heinzelmann J, Leidinger P, Backes C, Meese E, et al: Characterization of miR-146a and miR-155 in blood, tissue and cell lines of head and neck squamous cell carcinoma patients and their impact on cell proliferation and migration. J Cancer Res Clin Oncol: Nov 30, 2015 (Epub ahead of print).
26. Hou L, Chen J, Zheng Y and Wu C: Critical role of miR-155/ FoxO1/ROS axis in the regulation of non-small cell lung carcinomas. Tumour Biol: Nov 9, 2015 (Epub ahead of print).

27. You J, Yang H, Lai Y, Simon L, Au J and Burkart AL: ARID2, p110 $\alpha, \mathrm{p} 53$, and $\beta$-catenin protein expression in hepatocellular carcinoma and clinicopathologic implications. Hum Pathol 46: 1068-1077, 2015.

28. Raab JR, Resnick S and Magnuson T: Genome-wide transcriptional regulation mediated by biochemically distinct SWI/SNF complexes. PLoS Genet 11: e1005748, 2015.

29. Yu P, Wu D, You Y, Sun J, Lu L, Tan J and Bie P: miR-208-3p promotes hepatocellular carcinoma cell proliferation and invasion through regulating ARID2 expression. Exp Cell Res 336: 232-241, 2015. 